\title{
Improving shadows detection for solar radiation numerical models
}

\author{
F. Díaz*, H. Montero, D. Santana, G. Montero, E. Rodríguez, L. Mazorra Aguiar, \\ A. Oliver \\ Edificio Central del Parque Tecnológico, Institute for Intelligent Systems and Numerical Applications in Engineering, University of Las \\ Palmas de Gran Canaria, Campus de Tafira, 35017 Las Palmas de Gran Canaria, Spain
}

\section{A R T I C L E I N F O}

\section{Article history:}

Available online $\mathrm{xxx}$

\section{Keywords:}

Solar radiation

Shadows

Solar power

Adaptive meshes

\begin{abstract}
A B S T R A C T
Solar radiation numerical models need the implementation of an accurate method for determining cast shadows on the terrain or on solar collectors. The aim of this work is the development of a new methodology to detect the shadows on a particular terrain. The paper addresses the detection of self and cast shadows produced by the orography as well as those caused by clouds. The paper presents important enhancements on the methodology proposed by the authors in previous works, to detect the shadows caused by the orography. The domain is the terrain surface discretised using an adaptive mesh of triangles. A triangle of terrain will be under cast shadows when, looking at the mesh from the Sun, you can find another triangle that covers all or partially the first one. For each time step, all the triangles should be checked to see if there are cast or self shadows on it. The computational cost of this procedure eventually resulted unaffordable when dealing with complex topography such as that in Canary Islands thus, a new methodology was developed. This one includes a filtering system to identify which triangles are those likely to be shadowed. If there are no self shadowed triangles, the entire mesh will be illuminated and there will not be any shadows. Only triangles that have their backs towards the Sun will be able to cast shadows on other triangles. Detection of shadows generated by clouds is achieved by a shadow algorithm using satellite images. In this paper, Landsat 8 images have been used. The code was done in python programming language. Finally, the outputs of both approaches, shadows generated by the topography and generated by clouds, can be combined in one map. The whole problem has been tested in Gran Canaria and Tenerife Island (Canary Islands - Spain), and in the Tatra Mountains (Poland and Slovakia).
\end{abstract}

(c) 2017 Elsevier Inc. All rights reserved.

\section{Introduction}

As it is well known, renewable energies have increased their importance in power systems during the last decades. According to European Photovoltaic Industry Association [1], the global solar photovoltaic cumulative installed capacity for 2014, is 178 GW. This power is expected to be increased up to, around, 470 GW in 2019. Again, European Photovoltaic Industry Association [1] estimates that PV penetration in Europe in 2030 could be between $10 \%$ and $15 \%$ of the electricity demand, against the $3.5 \%$ of 2014 .

\footnotetext{
* Corresponding author.

E-mail address: felipe.diaz@ulpgc.es (F. Díaz).
} 


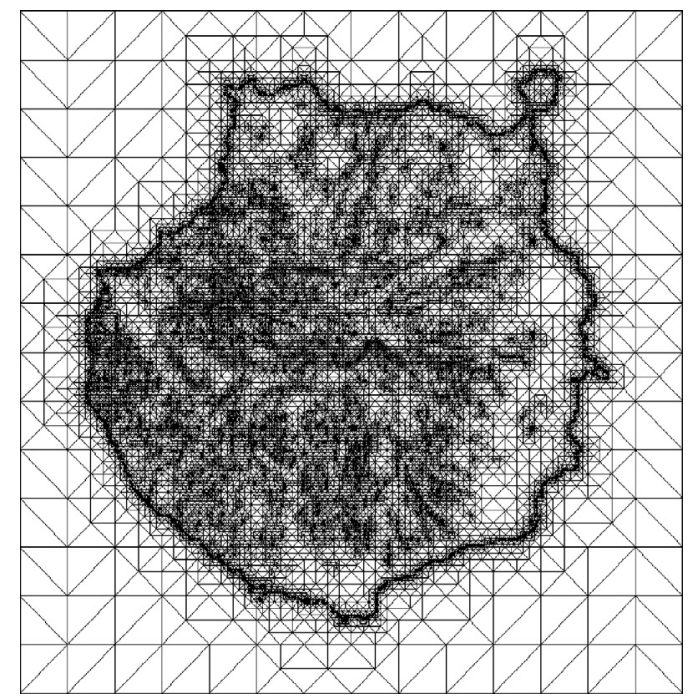

Fig. 1. Adaptive mesh for Gran Canaria Island.

The conclusion is clear, power systems will have to, increasingly, take into account solar power generated by means of photovoltaics or solar thermal electric facilities. In all of them the key parameter is solar radiation, either global or direct, and an accurate estimation of those variables needs a good knowledge about the spatiotemporal evolution of shadows over the whole analysis domain.

The solar radiation computation on the different parts of the terrain is based on the Šúri and Hofierka [2] model. This one uses geometrical, astrophysical and atmospheric considerations. In previous works of our research group we have introduced the use of triangle meshes adapted to both, elevation and albedo to analyse the solar radiation on the terrain $[3,4]$. This model has been applied in tasks of solar radiation and power forecasting too [5]. Shadows effects on surfaces have been studied by many authors because of its importance when estimating solar radiation [6,7]. In fact, we worked on a shadows model in [3], however its computational cost was quite high so a new methodology has been developed and presented in this paper. It includes a double filtering system to identify which triangles are those likely to be shadowed using adaptive meshes of triangles to describe the shapes of the terrain as solid surfaces. These ones can produce shadows because they are surfaces, not points.

In the other hand, the need of detecting the presence of clouds and their cast shadows, arises as an important problem to take into account, especially when you want to make a short term solar radiation forecasting. In this sense, clouds shadows detection is incorporated in the general shadows modelling, so that the new model greatly improves the predictive ability of our forecasting one. Clouds detection through satellite images has been studied by many authors. Statella and da Silva [8] proposed the application of Mathematical Morphology (MM) in shadow and clouds detection in high resolution images. Fisher [9] developed a method for masking clouds and shadows from clouds, in SPOT5 HRG Imagery. Semitransparent thin clouds detection can be done using Advanced Very High Resolution Radiometers (AVHRR) and Moderate Resolution Imaging Spectroradiometers (MODIS) [10]. Some researchers have analysed the use of the Landsat dataset [11] for these purposes, and this imagery will be used by the authors in this paper.

\section{Adaptive mesh for topography and albedo}

The chacterisation of the topography and the albedo is done using an adaptive method for refining and derefining a triangular mesh [3,4]. We will obtain a 2D mesh adapted to the altitude and albedo (if needed) variability. The first step is building a regular mesh $\tau_{1}$ of the domain starting from the Digital Elevation Map (DEM). Then, a sequence of nested meshes $\Gamma=\left\{\tau_{1}<\tau_{2}<\cdots<\tau_{m}\right\}$ will be obtained in a way that level $\tau_{j}$ is built through a global refinement of the previous level $\tau_{j-1}$ with the 4-T Rivara's algorithm [12]. When the refinement is done, a new sequence $\Gamma^{\prime}=\left\{\tau_{1}<\tau_{2}^{\prime}<\cdots<\tau_{m^{\prime}}^{\prime}\right\}, m^{\prime} \leq m$, is defined derefining the mesh [13]. In case that we want a mesh adapted to both surface (topography) and albedo, two derefinement parameters are needed, one for elevation, $\varepsilon_{h}$, and one for albedo, $\varepsilon_{a}$. These two parameters will give the accuracy of the mesh.

Once the process is finished, we obtain an adaptive mesh that characterise the topography and the albedo, if necessary. Fig. 1 shows a fine adaptive mesh for Gran Canaria Island adapted to topography. To get good results for solar radiation purposes, a coarser mesh is needed. In fact, a mesh with 5866 nodes and 11,683 triangles is enough for Gran Canaria Island (Canary Islands - Spain), which has an area of $1560 \mathrm{~km}^{2}$.

Using the same methodology we have studied a very different place, the Tatra Mountains placed between Poland and Slovakia. These mountains are part of the Carpathian Mountains, and have an area of $785 \mathrm{~km}^{2}$. The whole landscape 


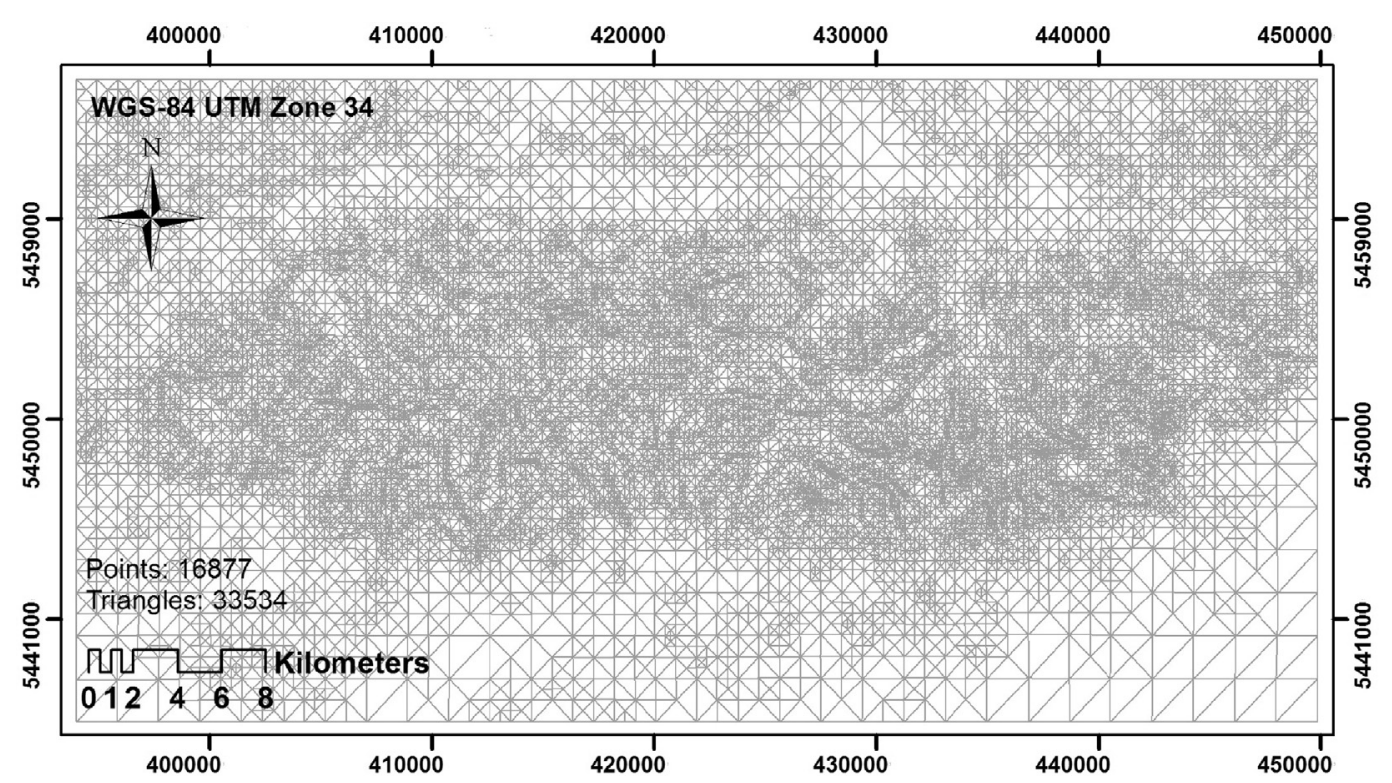

Fig. 2. Adaptive mesh for the Tatra Mountains.

constitutes the Tatra National Park, really two national parks, one in Poland and one in Slovakia. It has a lot of mountain peaks, glacial cirques, deep valleys etc. Fig. 2 shows a fine mesh for these mountains. Again, for our purposes, a mesh with 6284 nodes and 12,431 triangles is enough.

\section{Solar radiation model}

Whether you want to characterise solar radiation in an area, as if you want to predict it, the procedure would be to compute the clear sky radiation values on each triangle of the adaptive mesh and, after that, to compute the real sky radiation values as a correction of the previous one, using the clear sky index [3-5]. The global solar irradiance is the addition of three components: beam, diffuse and reflected. The beam irradiance is not reflected or scattered and reaches the surface directly. The diffuse irradiance is the scattered one that goes in all directions. The reflected irradiance or albedo irradiance, is the one reflected from a surface to other. Since this model has been discussed in the literature [2-5], we will only show the equations of the different components of the clear sky radiation for a horizontal surface, in order to know the influence of the shadows in them.

The beam component is:

$$
G_{b c}(0)=G_{b 0 c} L_{f} \sin h_{0}
$$

As can be seen, the beam radiation depends on the solar altitude angle, $h_{0}$ and on the light factor, $L_{f}$, which takes into account if the surface is sunlit or shadowed. For sunlit surfaces, $L_{f}=1$, and for absolutely shadowed ones, $L_{f}=0$, so $0<L_{f}$ $<1$. Moreover, we can compute the light factor as $L_{f}=L_{f s s} \cdot L_{f c s}$, where $L_{f s s}$ is the light factor for self shadows, this is, the shadows projected by a surface over itself (they are backwards to the Sun), and $L_{f c s}$ is the factor for the shadows projected by a different surface.

The diffuse irradiance is estimated using:

$$
G_{d c_{e}}(0)=G_{0} T_{n}\left(T_{L K}\right) F_{d}\left(h_{0}\right)
$$

The diffuse component on an inclined surface, $G_{d c}(\beta)$, with $\beta$ the angle between the normal to a triangle and the horizontal plane, is computed taking into account if the surface is shadowed or not, according to Muneer [14]. For shadowed ones,

$$
G_{d c}(\beta)=G_{d c_{e}}(0) F(\beta)
$$

$$
G_{d c}(0)=G_{d c_{e}}(0)
$$

where $F(\beta)$ is:

$$
F(\beta)=r_{i}(\beta)+N_{d}\left(K_{b}\right)\left[\sin \beta-\beta \cos \beta-\pi \sin ^{2} \frac{\beta}{2}\right]
$$




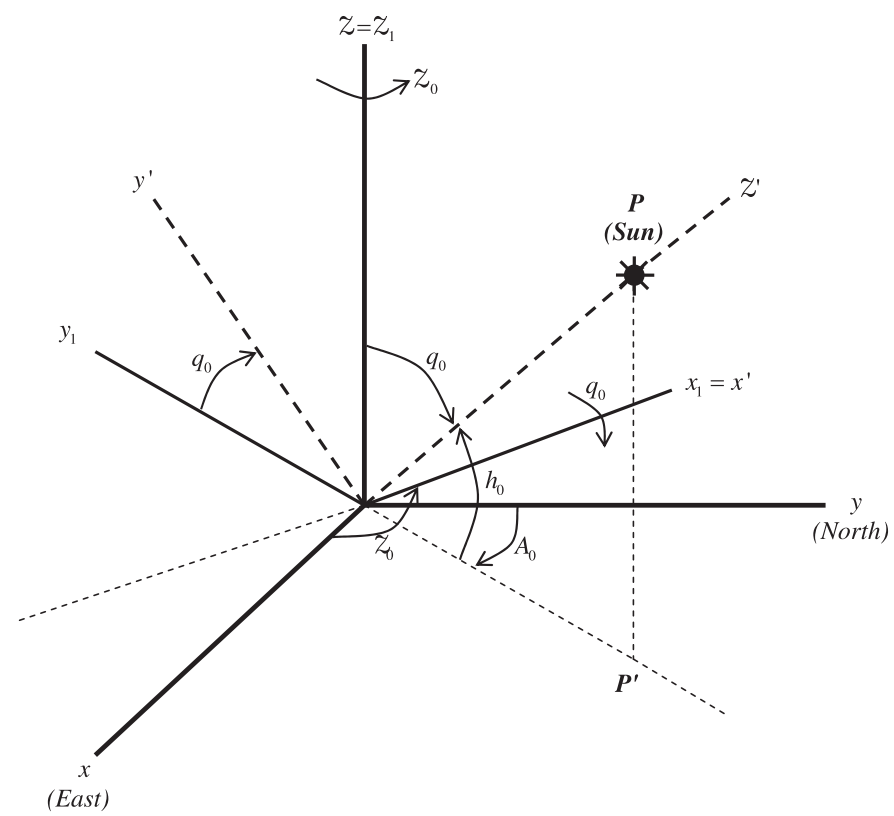

Fig. 3. Reference systems.

$N_{d}\left(K_{b}\right)$ is computed using:

$$
\begin{aligned}
& \text { IF } L_{f s s} \cdot L_{f c s}=L_{f}=1 ; \quad N_{d}\left(K_{b}\right)=0,00263-0,712 K_{b}-0,6883 K_{b}^{2} \\
& \text { IF } L_{f s s} \cdot L_{f c s}=L_{f} \neq 1 ; \quad N_{d}\left(K_{b}\right)=0,25227
\end{aligned}
$$

Where $K_{b}$ measures the quantity of available beam irradiance as a ratio between the horizontal beam irradiance and the extraterrestrial horizontal one, $G_{0}(0)$.

$$
K_{b}=G_{b c}(0) / G_{0}(0)
$$

As for the reflected irradiance, this one depends on the global horizontal irradiance $G_{c}(0)$, the mean ground albedo $\rho_{g}$ and a fraction of the ground viewed by an inclined surface $r_{g}(\beta)$ [15].

$$
G_{r}(\beta)=\rho_{g} G_{c}(0) r_{g}(\beta)
$$

where

$$
\begin{aligned}
& r_{g}(\beta)=(1-\cos \beta) / 2 \\
& G_{c}(0)=G_{b c}(0)+G_{d c}(0)
\end{aligned}
$$

So, it is clear that the presence of shadows or not affects the solar radiation values in the model, diminishing it or not. So the main problem to solve now, for an accurate estimation of topographic shadows, is the computation of the light factor, $L_{f}$.

\section{Detection of topographic shadows}

\subsection{General procedure}

The general technique to find which triangles from the mesh are shadowed is described in [3,4]. In short, the solution to this geometrical problem is finding those triangles that, looking at the mesh from the Sun, have at least another triangle that covers the former. To do that, a new reference system $x^{\prime}, y^{\prime}$ and $z^{\prime}$, with $z^{\prime}$ in the direction of the beam radiation (see Fig. 3), is constructed. Then, the mesh is projected on the plane $x^{\prime} y^{\prime}$. This way we can observe what "the Sun sees" for each time step, and find out what triangles are overlapped, if any (Fig. 4).

Of course, the position of the Sun for every time step must be known. There are many developed algorithms to determine this position. In this work, the PSA algorithm [16] is used. Starting from the variables, solar azimuth $A_{0}$ and solar altitude 

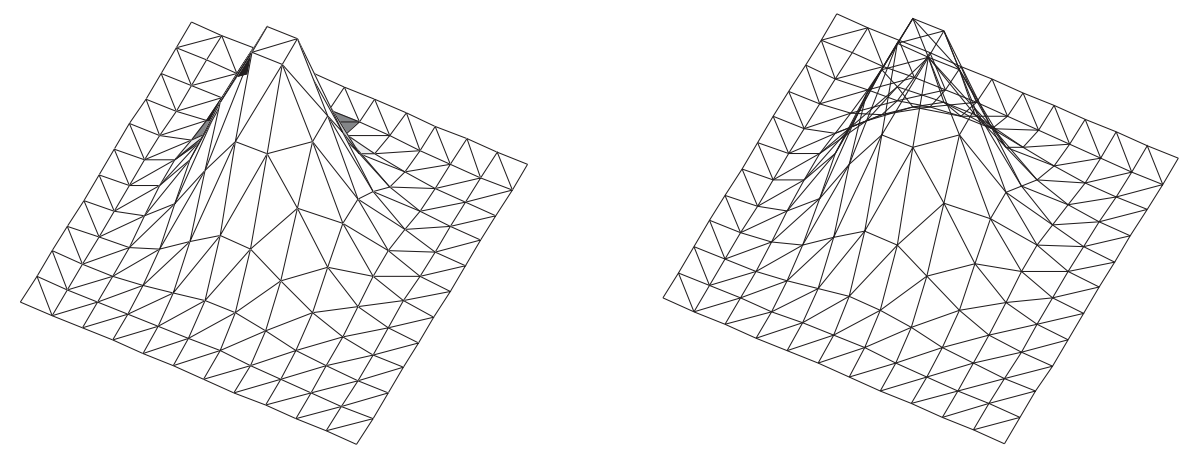

Fig. 4. Mesh from the Sun and projected.
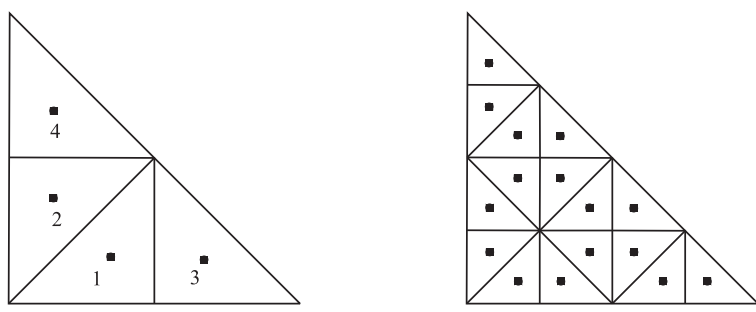

Fig. 5. Warning points.

$h_{0}$ (Fig. 3), we make a coordinate transformation to get $z^{\prime}$ in the beam radiation direction according to PSA algorithm. Now that we have a "view from the Sun", it is easy to determine the overlapping among mesh triangles.

Studying for each time step the intersected triangles, and making a temporal refinement for each analysed one of them, a level of shading is assigned to every shadowed triangle according to the number of warning points that are found inside other triangles (see Fig. 5). This is the so called light factor, $L_{f}$, which was already used in Section 3.

The problem in this technique arises when you want to apply it to a whole domain like the island of Gran Canaria (Fig. 1) because the computational cost is too high, making the process too slow for some purposes.

\subsection{Triangles filtering and final computation}

Because of that, a new technique was developed, based in the previous filtering of the candidate triangles, before checking the final overlapping in the mesh. When will a triangle be shadowed? It may be shadowed due either to own or self shadows, either the shadows cast by other triangle. The self shadows are those produced by a surface over itself, in example, when they are back to the Sun. This is easy to be determined working with the incidence angle ${ }^{1}\left(\delta_{\text {exp }}\right)$ as its value should be greater than $\pi / 2$ (see Fig. 6 ).

We have defined a Self Shadows Light Factor $\left(L_{f s s}\right)$, with the values:

$$
0 \quad \text { IF }\left|\delta_{\text {exp }}\right|>(\pi / 2)
$$

$$
1 \quad \text { IF }(\pi / 2) \geq\left|\delta_{\text {exp }}\right| \geq 0
$$

As the analysis of the cast shadows is the one that needs more computational effort, the first thing to do is avoid analysing all those back to Sun triangles (self shadowed) in search of cast shadows. It is very helpful filtering the triangles according to:

1. If there are no self shadowed triangles (back to Sun), the whole mesh is illuminated and there are no shadow at all.

2. Only back to Sun triangles are able to cast shadows. We called them potential 1 triangles (see Fig. 7).

Now, each triangle $\Delta$ is analysed, in the search of other triangle $\Delta^{\prime}$ that intersects $\Delta$ and is before him, this is, coordinate $z^{\prime}$ of the intersection point is greater for $\Delta^{\prime}$ than for $\Delta$. To carry out this process computationally efficiently, the triangles will be filtered according to their ability for casting shadows. The process begins partitioning the mesh in the plane $x^{\prime}-y^{\prime}$ and projecting it towards $z^{\prime}$ forming parallelepipeds that we have called cubes (see Fig. 8). Cubes are variable in size and direction according to the computed time step. The lower the Sun is, early in the morning and late in the evening, the more triangles will be covered by cubes.

\footnotetext{
1 Angle between the solar vector and the normal to a triangle surface.
} 


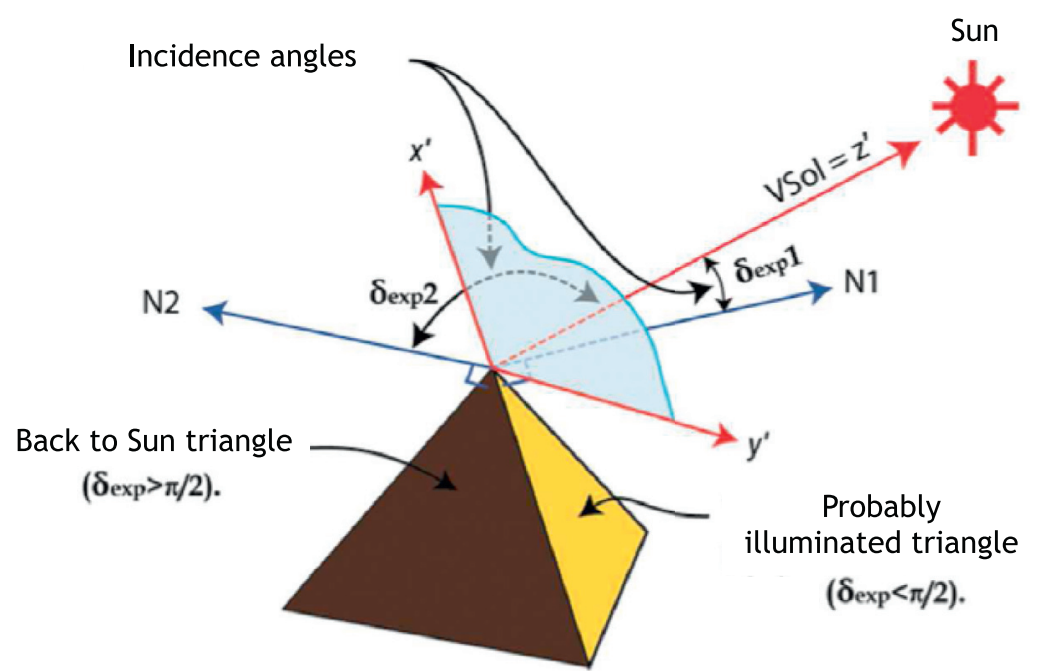

Fig. 6. Incidence angle and self shadows.

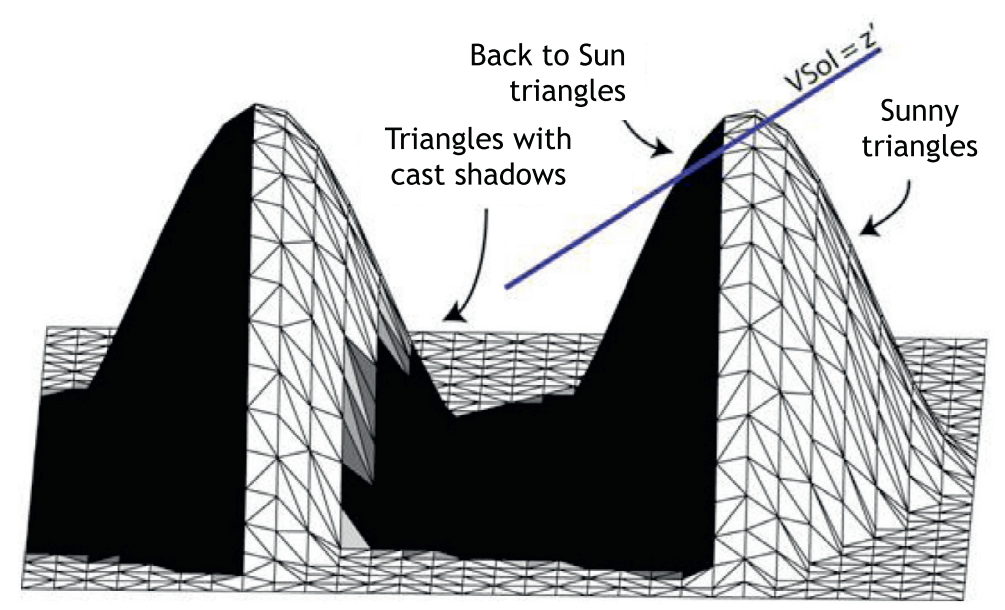

Fig. 7. Cast and self shadows.

The dimensions of these parallelepipeds in the plane $x^{\prime}-y^{\prime}$ are such that the squares forming their bases should measure (their sides), the maximum existent distance from the centre to each of its nodes, of all triangles of the mesh. This way, in the worst case, when studying the mesh largest triangle, its nodes does not extend beyond the eight cubes that surround the cube containing its centre.

Each potential 1 triangle is associated to a cube containing it by the rotated coordinates of its centre of gravity. Thus, by studying what other triangles are in the environment of any back to Sun triangle, you do not have to search the entire grid, but only on cubes that are immediately around them. Once all potential 1 triangles are classified in cubes, we proceed to evaluate each of the triangles which are illuminated, to see if there is a triangle that is, in projection, ahead of it partially or completely and thus, be able to assess how much shade it receives.

Each of the illuminated triangles is analysed iteratively. Firstly, all potential 1 triangles which are not in the immediate environment of the studied triangle, are discarded. These potential 1 triangles, close to the analysed one, are called potential 2 triangles (back to the sun and also located in close cubes) (see Fig. 9). Once the potential 2 triangles are identified, these ones and the studied triangle are embedded into cubic domains as tight as possible. Those potential 2 triangles which cubic domain intersects the analysed triangle new domain, and are also closest to the Sun, are the triangles that are more likely to cast shadows on the studied triangle. These ones are called potential 3 triangles (back to Sun, in close cubes, and with intersected domains). The rest of potential 2 triangles are discarded because they are not able to cast shadows on the analysed triangle (see Fig. 10).

The final shading study is carried out checking the projection of shadows on a small set of points which are called warning points (wp). The $w p$ are a set of internal points of the triangle evenly distributed following the 4-T Rivara 


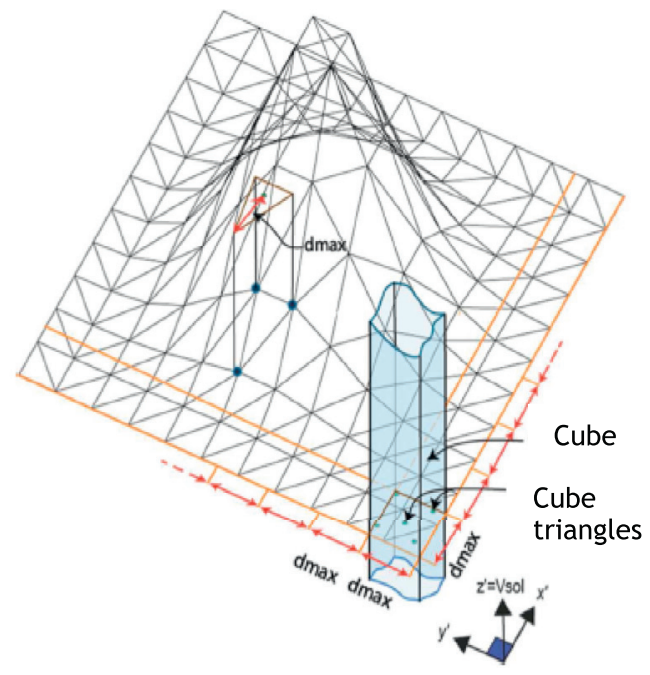

Mid day

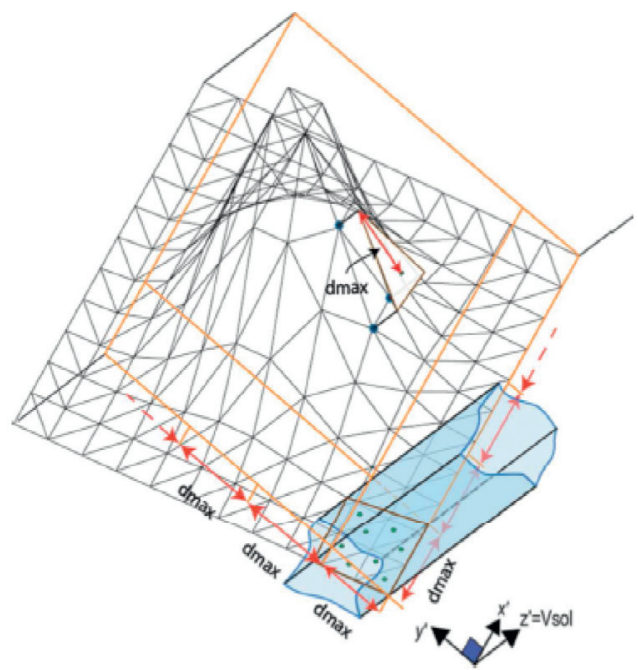

Morning

Fig. 8. Cubes formation.

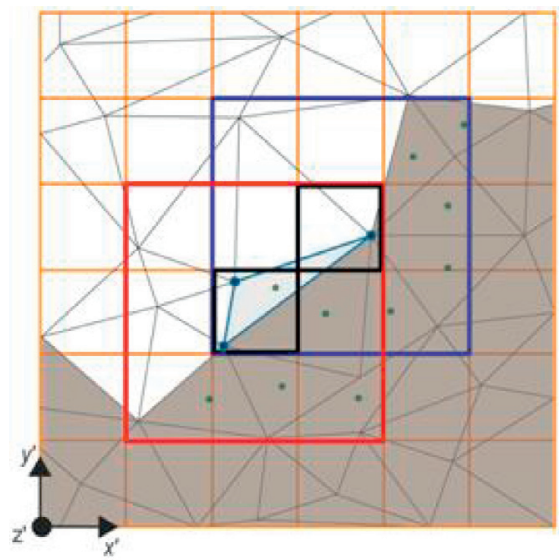

Analysed triangle

Cubes that include the triangle

Cubes inmediate surroundings

Illuminated triangles

Potential 1 triangles (back)

Potential 2 triangles (back and close)

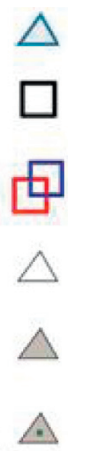

Fig. 9. Envelope and potential 2 triangles.

algorithm [12] and that coincide with the geometric centres, outcome of each triangle subdivision (see Fig. 5). A warning point will be shaded when (Fig. 11):

1. The warning point, projected on the plane $x^{\prime}-y^{\prime}$, should be into the projection of any potential 3 triangle.

2. The warning point should be farther from the Sun than point $q$ in the potential triangle, this is, its coordinate $z^{\prime}$ must be minor than that from point $q$.

The cast shadows light factor, $L_{f c s}$ is computed as:

$$
L_{f c s}=\frac{n_{w p}-i}{n_{w p}}
$$

where $i=0,1, \ldots, n_{w p}$ is the number of warning points which are shadowed, and $n_{w p}$ the number of warning points. The final light factor, $L_{f}$ is

$$
L_{f}=L_{f c s} \cdot L_{f s s}
$$

\section{Clouds shadows}

To study the shadows generated by clouds on the terrain, we will use satellite images. In this case, LANDSAT- 8 images are used. Imagery comes from two sensors: 


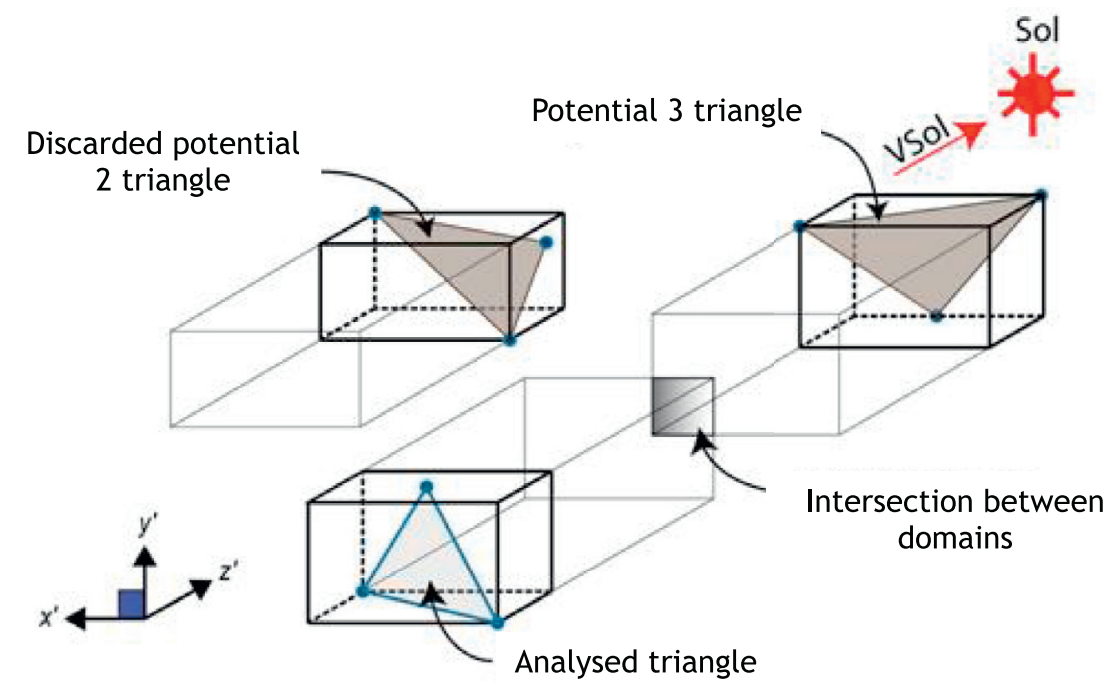

Fig. 10. Cubic domains.

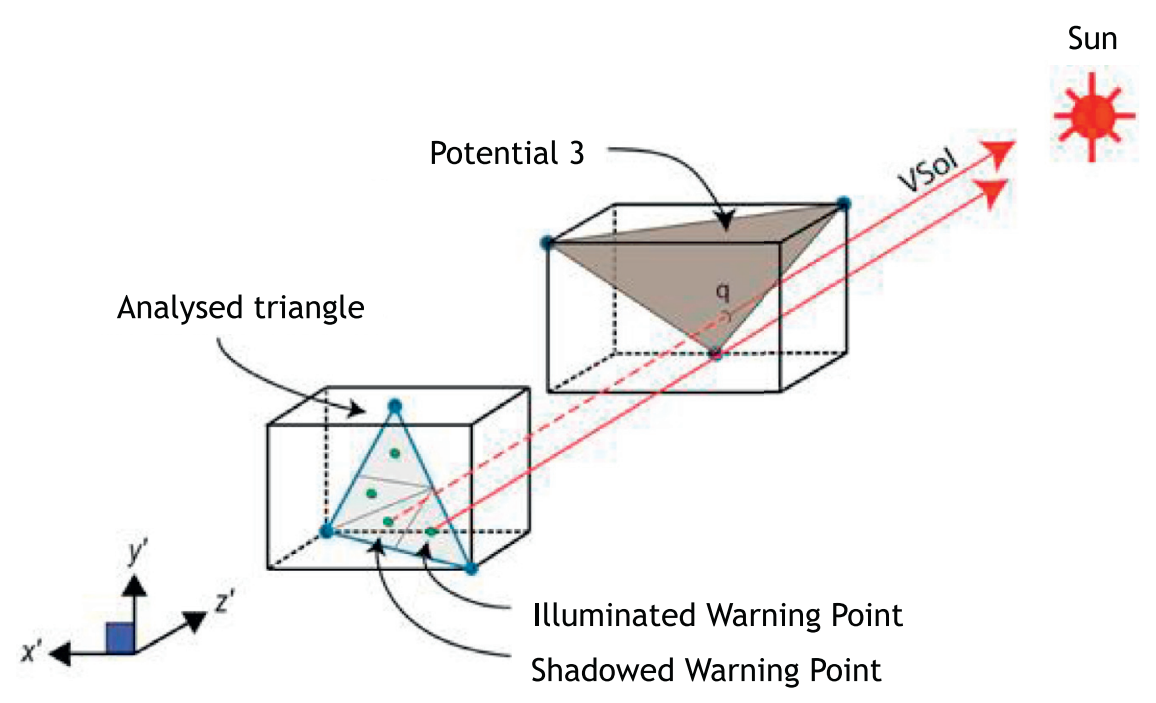

Fig. 11. Warning points analysis.

- Operational Land Imager (OLI)

- Thermal Infrared Sensor (TIRS) (bands 10 and 11)

LANDSAT-8 images obtained by sensors (OLI) and (TIRS) consist of nine spectral bands with a spatial resolution of $30 \mathrm{~m}$ for bands $1-7$ and 9 . The resolution for the panchromatic band (8), is $15 \mathrm{~m}$. The two thermal bands 10 and 11 are very interesting for surface temperatures, and are taken with a resolution of $100 \mathrm{~m}$.

In the other hand, LANDSAT- 8 offers a very interesting 16 bits Quality Control Band with additional data. The presence of water, ice or snow, cirrus and/or clouds, are determined with different levels of confidence given by two bits (No confidence; low; intermediate; high).

The methodology is explained through an example developed on images from Tatra Mountains (see Fig. 2). Our interest in focusing on detection of clouds, cirrus and shadows, leads us to make some RGB compositions. To detect clouds shadows on the terrain surface, we can use some OLI bands compositions like those shown in Figs. 12 and 13.

The clouds detection method applied, is based on the use of the quality bands of the satellite [17]. It is possible to mask cirrus and/or clouds with different levels of confidence. We applied High (65-100\%) and Intermediate/High level (33-100\%). The process for estimating cast shadows from clouds begins with the obtention of the radiance values from the image and then converting these ones into brightness temperature and, finally, compute the actual temperature, which is used to estimate clouds altitude. Cast shadows are defined using the estimated shadow length (see Fig. 14). 


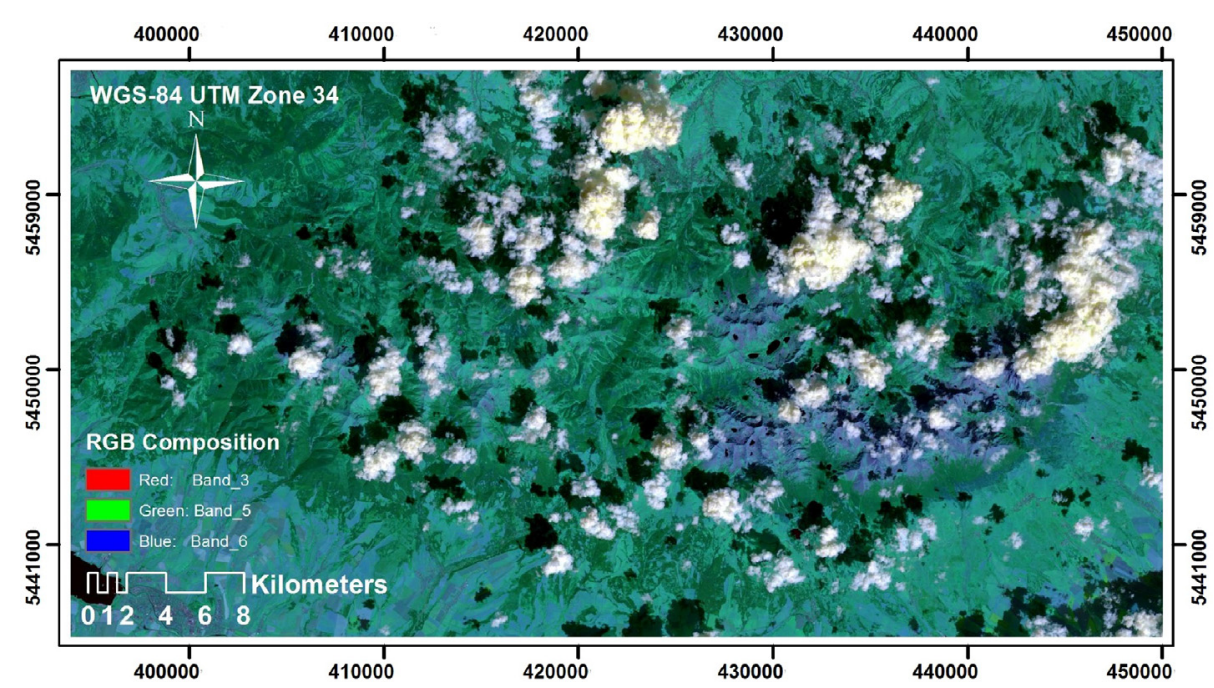

Fig. 12. RGB Composition. Bands $3,5,6$.

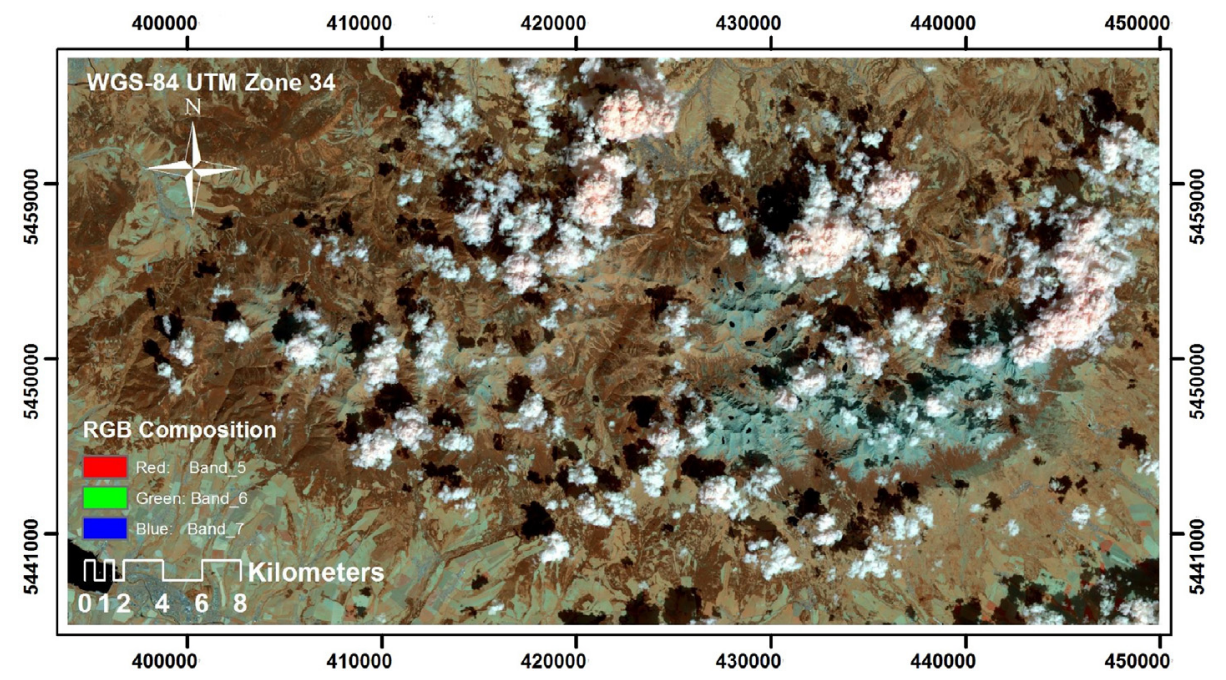

Fig. 13. RGB Composition. Bands 5, 6, 7.

According to Zanter [17] data from OLI and TIRS sensors can be rescaled to the radiance values in the top of the atmosphere, using Eq. (16).

$$
L_{\lambda}=M_{L} \cdot Q_{c a l}+A_{L}
$$

where $L_{\lambda}$ is the spectral radiance at the top of the atmosphere (TOA) $\left(\mathrm{W} /\left(\mathrm{m}^{2} \cdot \operatorname{srad} \cdot \mathrm{um}\right)\right), M_{L}$ is the multiplicative scaling factor, $A_{L}$ is the additive scaling factor, and $Q_{c a l}$ is the standard product quantified and calibrated pixel values.

Brightness temperature (in Kelvin) [17], is computed according to:

$$
T_{b}=\frac{K_{2}}{\ln \left(\frac{K_{1}}{L_{\lambda}}+1\right)}
$$

where $T_{b}$ is the apparent brightness temperature (K), $K_{1}$ and $K_{2}$ are specific conversion constants for bands 10 and 11 .

The Real Land Surface Temperature is estimated using Eq. (18):

$$
T_{s}=c_{0}+T_{i}+c_{1} \cdot\left(T_{i}-T_{j}\right)+2 c_{2} \cdot\left(T_{i}-T_{j}\right)+\left(c_{3}+w c_{4}\right) \cdot(1-\varepsilon)+\left(c_{5}+w c_{6}\right) \cdot \Delta \varepsilon
$$

where coefficients are estimated according to Jiménez-Muñoz et al. [18] and Rajeshwari and Mani [19].

According to Yost et al. [20] and Inoue [21], the Brightness Cloud Temperature determined from the thermal bands is assumed to be the Real Cloud Temperature. Each cloud, considered as a group of pixels, is assigned an average temperature to determine its altitude, which is computed by Henning's formula (Eq. (20)), according to Chiyu et al. [22].

$$
H(m)=125 \cdot\left(T-T_{d}\right)
$$




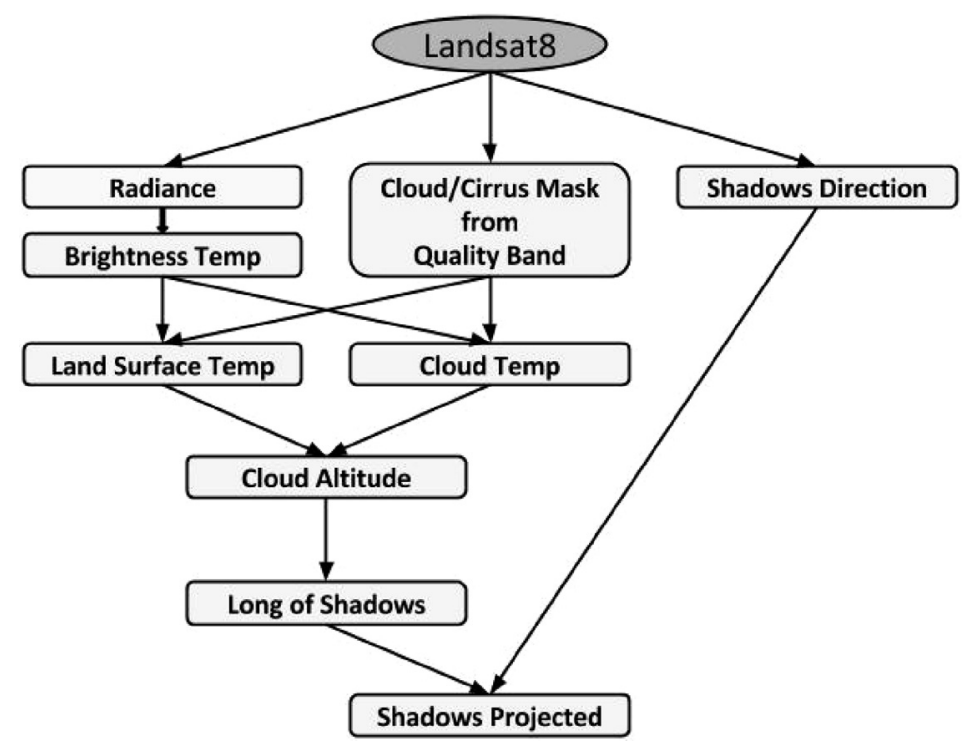

Fig. 14. Shadows from clouds flowchart.

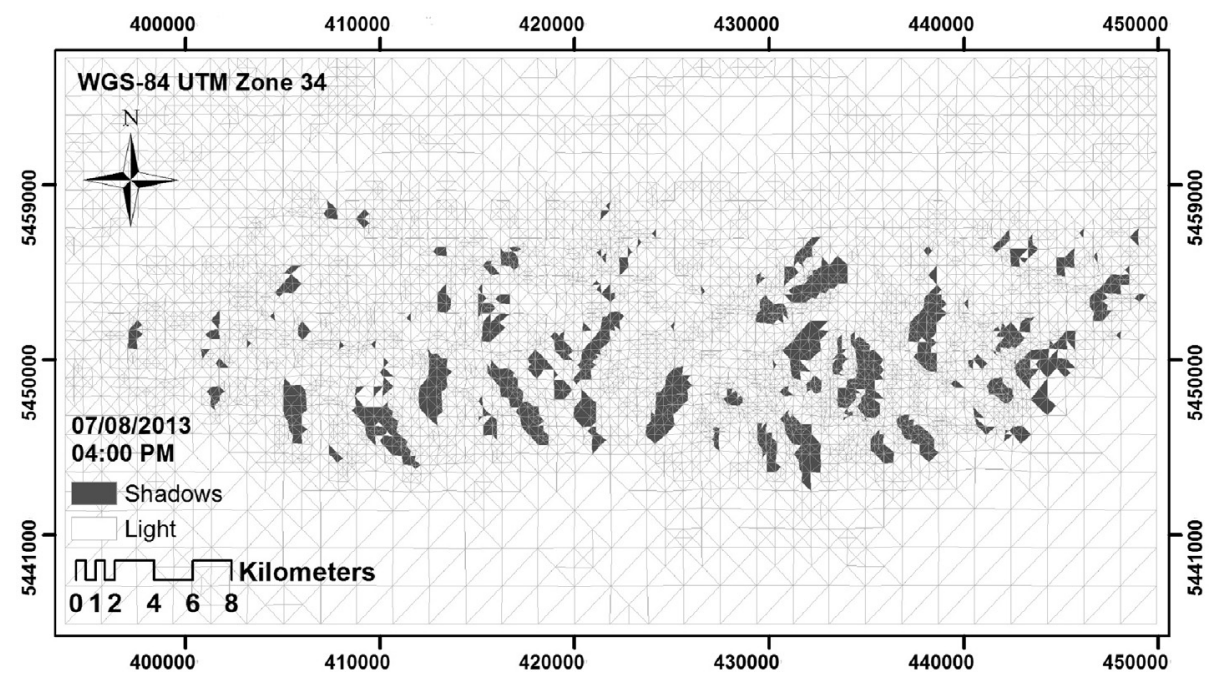

Fig. 15. Topographic shadows on Tatra Mountains. August, 16:00 UTC.

where $T$ is the surface temperature, $T_{d}$ is the clouds temperature. Once the clouds altitude $(H)$ is known, determining the shadows length $(L)$ is easy $[23,24]$ :

$$
L=\frac{H}{\tan h_{0}}
$$

where $h_{0}$ is the elevation angle (see Fig. 3).

\section{Simulation results}

Simulations have been performed on Gran Canaria and Tenerife Island (Canary Islands - Spain), and on the Tatra Mountains (Poland and Slovakia).

\subsection{Topographic shadows results}

Topographic shadows simulations have been performed on all the studied domains. In example, for the Tatra Mountains domain, on an arbitrary date (16:00 UTC, August, 7th), the shadows shown in Fig. 15 will be obtained. 


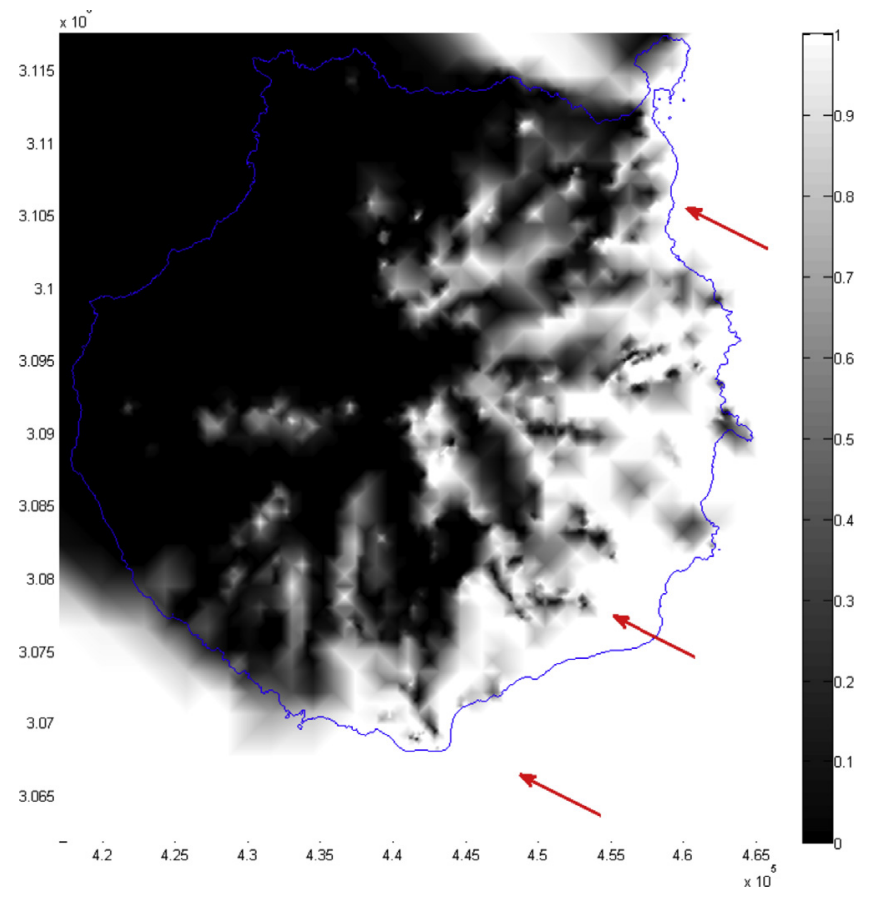

Fig. 16. Topographic shadows on Gran Canaria Island. January, 1st, 8:00 UTC.

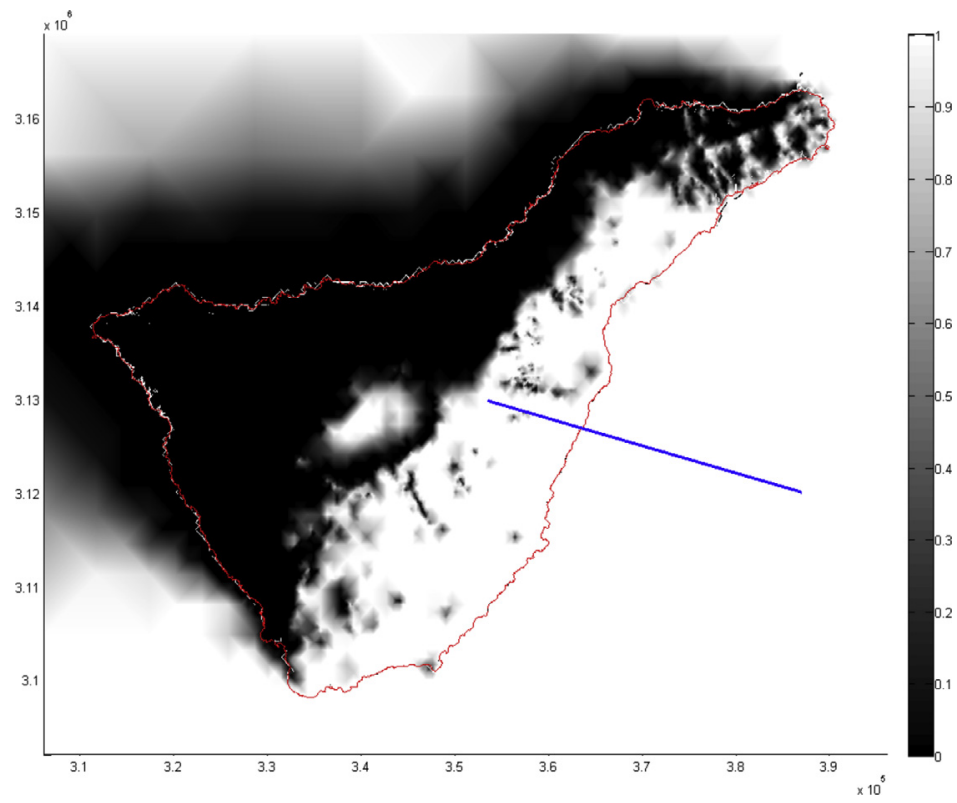

Fig. 17. Topographic shadows on Tenerife. February, 15th, 8:00 UTC. (For interpretation of the references to colour in this figure, the reader is referred to the web version of this article.)

As for the shadows analysis in the Canary Islands, here we show two examples, one for Gran Canaria (Fig. 16), and one for Tenerife (Fig. 17). Vectors (in blue or red) in these figures represent the solar vector in the exact time and date of the simulation. The grey scale, from 0 to 1 represents the quantity of shadow in a particular place according to the number of relative shadowed warning points $\left(L_{f}\right)$. Of course, these images have been softened to reach a more realistic approach.

In Fig. 18, a three-dimensional view of the shadows affecting Tenerife at 8:00 UTC for February, 15th, can be seen. Please note the details of light and shadows around the Mount Teide (3718 m altitude), and its surrounding Las Cañadas caldera. Moreover the graphical aspect that we can get, each point of the studied surface belongs to a mesh triangle, which is 


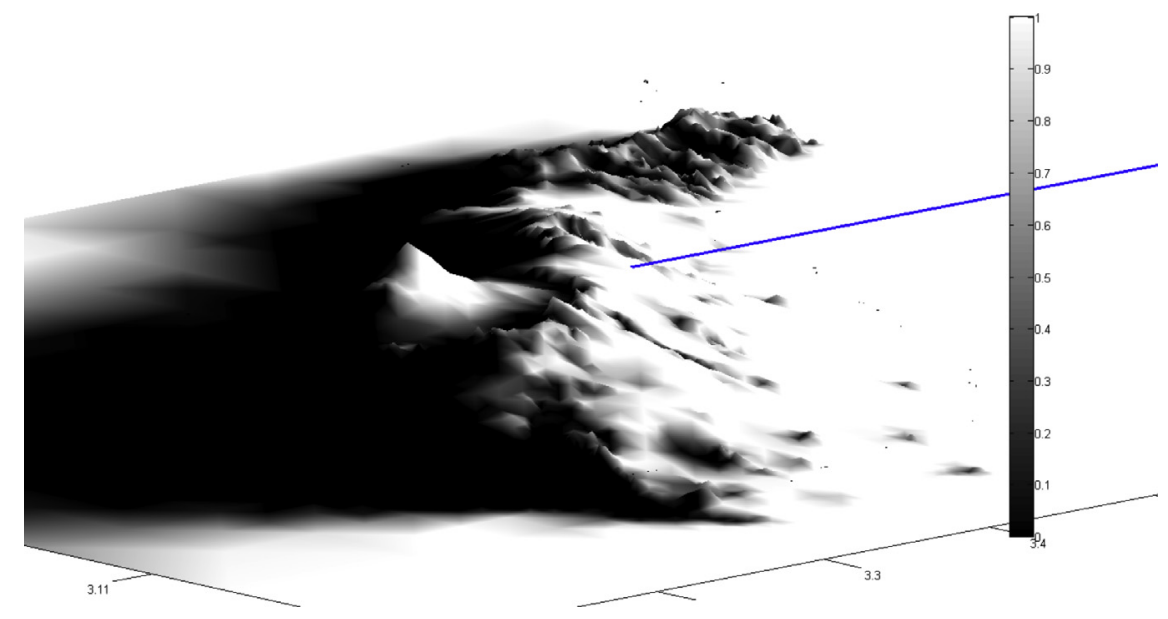

Fig. 18. 3D topographic shadows on Tenerife. February, 15th, 8:00 UTC.

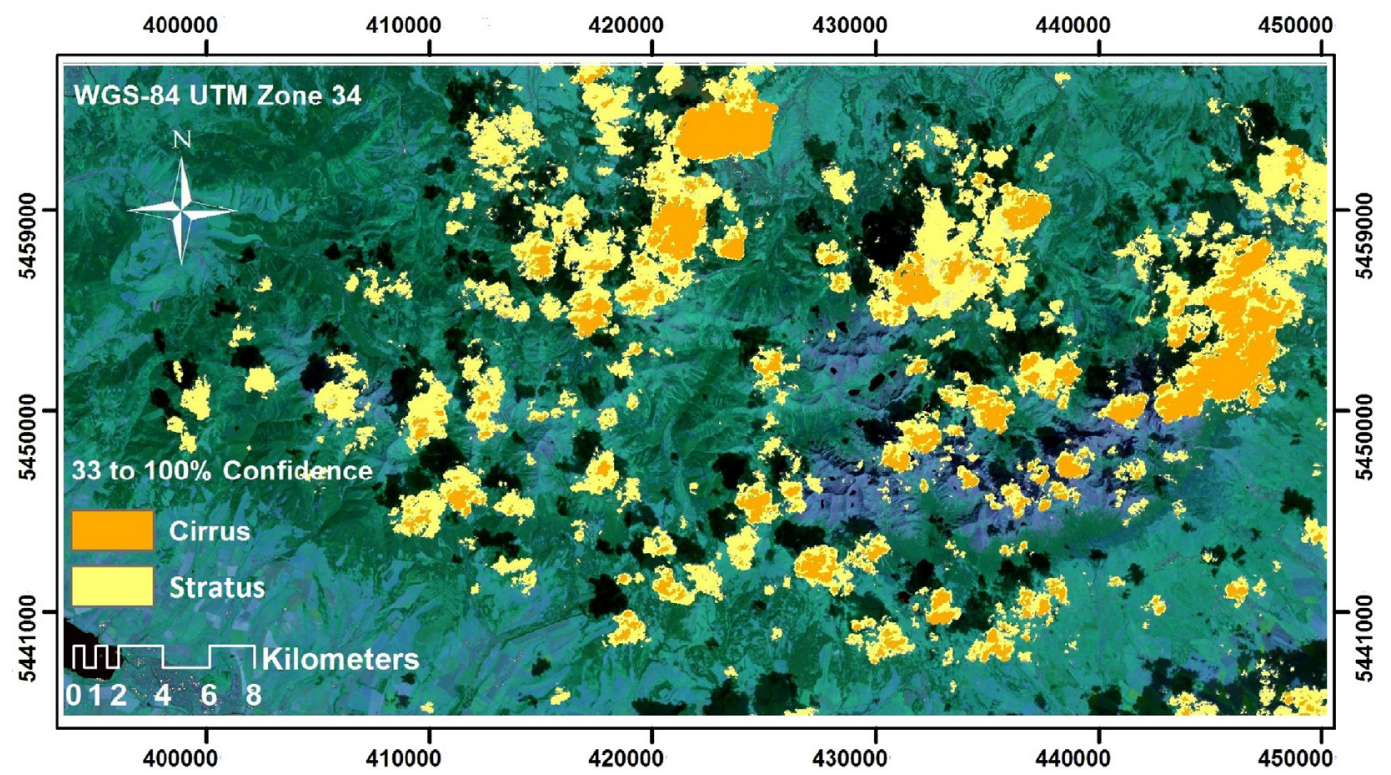

Fig. 19. Mask for clouds and cirrus. $33-100 \%$ confidence.

assigned a light factor, $L_{f}$, that allows us to take into account the quantity of self or cast shadows that a surface suffers. The same can be done over a triangle or more, representing not terrain but a solar collector.

\subsection{Clouds shadows results}

Simulations for detecting and analysing shadows produced by clouds, have been performed using LANDSAT-8 satellite images for the Tatra Mountain region, between Poland and Slovakia. Firstly, clouds and cirrus are detected taking advantage from the quality band information. Minimum differences were found between the work done with a mask of High Confidence (66-100\%), and that done with Medium and High Confidence (33-100\%). Fig. 19 shows the mask for clouds (mainly stratus, this is, low cloudiness) and cirrus (high clouds), with medium and high confidence, $33-100 \%$. Please note how cirrus are masked inside clouds representing the highest parts of these ones as was said before.

Applying what was explained in Section 5, we can estimate the shadows mean length, and the clouds altitude. The mean Euclidean length of the cast shadows is $1472 \mathrm{~m}$, and the mean altitude of the clouds is $2026 \mathrm{~m}$. These clouds are, therefore, stratus, stratocumulus and/or nimbostratus.

In the other hand, Table 1 shows the average land surface and cloud temperature estimated for bands 10 and 11 . Table 2 shows the mean cloud altitude estimated from the temperature variation.

Using bands 10 and 11, a shadows mask can be done (see Figs. 20 and 21). 
F. Díaz et al./Applied Mathematics and Computation 000 (2017) 1-15

Table 1

Land surface and clouds temperature.

\begin{tabular}{ll}
\hline Mask & Value (K) \\
\hline Mean land surface temp. & 301 \\
Mean cloud temp. band 10 & 278 \\
Mean cloud temp. band 11 & 285 \\
\hline
\end{tabular}

Table 2

Mean cloud altitude and mean longitude of shadow projections.

\begin{tabular}{lll}
\hline Band & Altitude $(\mathbf{m})$ & Longitude $(\mathbf{m})$ \\
\hline Band 10 & 2881 & 2067 \\
Band 11 & 2006 & 1439 \\
\hline
\end{tabular}

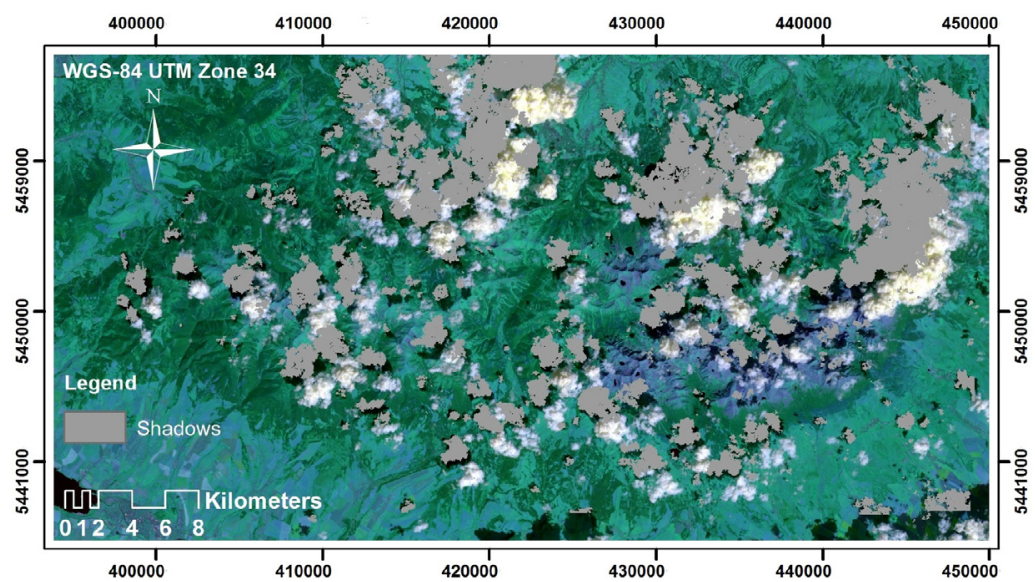

Fig. 20. Shadows mask. Band 10.

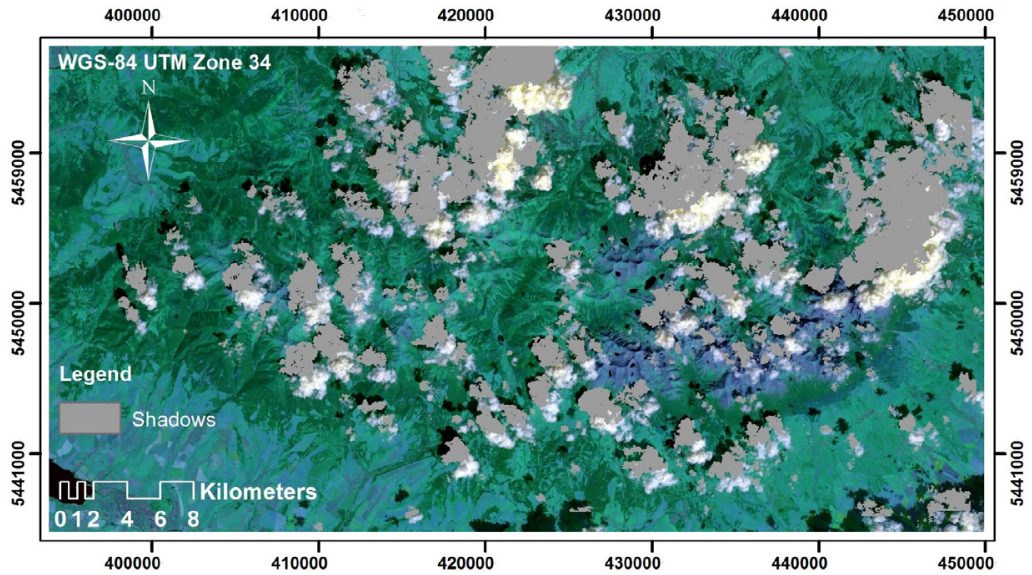

Fig. 21. Shadows mask. Band 11.

It seems that shadow mask for band 10 fits better for low altitudes while in higher locations, the shadows were overprojected. Opposite behaviour was found in the shadow mask for band 11.

Finally, we will find which triangles are affected by shadows from clouds, simply moving the mask to the mesh. Fig. 22 shows the mesh affected of these new shadows, computing the Light Factor for each triangle as said before, taking into account the number of shaded warning points.

Fig. 23 shows, both, the shadows on the mesh and their actual shape showing the shadows real borderline. 


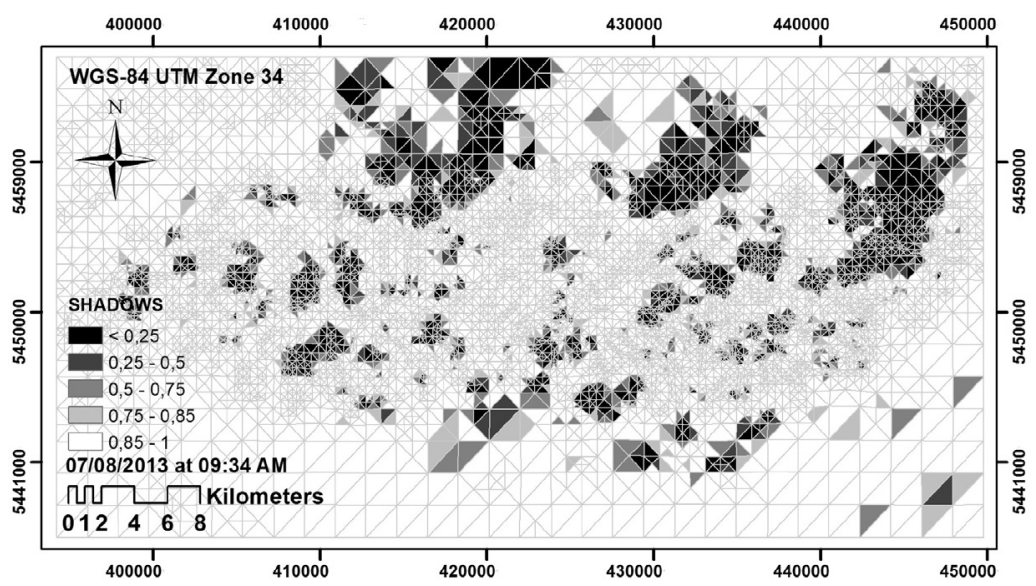

Fig. 22. Shadows from band 10 into mesh.

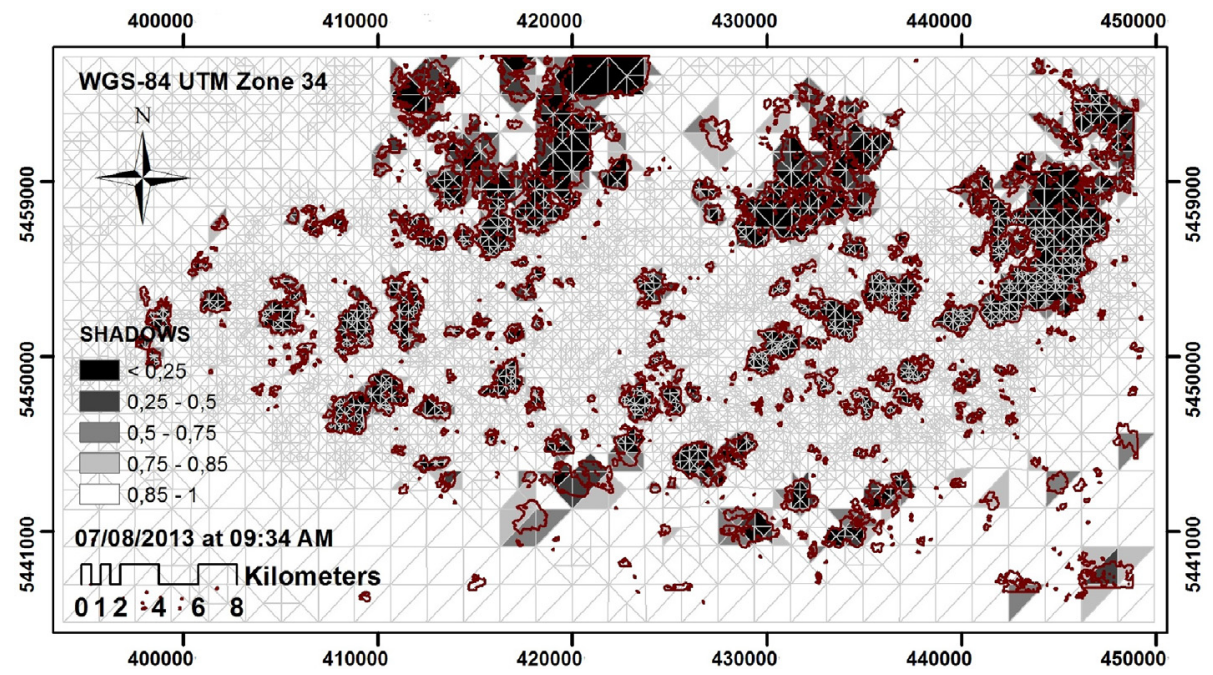

Fig. 23. Shadows borderline from band 10 into mesh.

\section{Conclusions}

In this paper, a new method for detecting shadows from topography has been developed. The adaptive triangulation related to the topography and albedo (when necessary), is essential in order to obtain accurate results of shadow distribution and solar radiation. Due to the high computational need of the process, we have developed a method that includes adaptive meshes and a 3 filtering way that leads to a low computational cost for detecting shadows.

In the other hand, the inclusion of shadows generated by clouds from satellite images into the mesh, increases the reliability of the solar model estimation. Obviously, the accuracy of the shadows estimation depends on the triangle size. Inaccuracies to estimate shadows from clouds appear due to:

1. Assumption that real temperature for clouds are equal to its brightness temperature

2. No correlation was applied between land surface temperature and altitude

3. Rough estimation of cloud altitude by using Henning's formula

Future research includes improving the method avoiding the above mentioned inaccuracies, the integration of cloud motion vectors analysis in the model to estimate clouds evolution, or integration of the model with analysis of whole sky camera images. Anyway, the objective would be to develop a nowcasting predictive solar code using the estimation of clouds shadows detection. 


\section{Acknowledgements}

This work has been supported by the Ministerio de Economía y Competitividad, Spanish Government, Grant contract CTM2014-55014-C3-1-R.

\section{References}

[1] European Photovoltaic Industry Association, Global Market Outlook for Photovoltaics 2015-2019, European Photovoltaic Industry Association, 2015. Technical Report

[2] M. Šúri, J. Hofierka, A new GIS-based solar radiation model and its application to photovoltaic assessments, Trans. GIS 8 (2) (2004) $170-175$.

[3] G. Montero, J.M. Escobar, E. Rodríguez, R. Montenegro, Solar radiation and shadow modelling with adaptive triangular meshes, Sol. Energy 83 (7) (2009) 998-1012.

[4] F. Díaz, G. Montero, J.M. Escobar, E. Rodríguez, R. Montenegro, An adaptive solar radiation numerical model, J. Comput. Appl. Math. 236 (18) (2012) 4611-4622.

[5] F. Díaz, G. Montero, J.M. Escobar, E. Rodríguez, R. Montenegro, A new predictive solar radiation numerical model, Appl. Math. Comput. 267 (2015) 596-603.

[6] A. Niewienda, F.D. Heidt, SOMBRERO: a PC-tool to calculate shadows on arbitrarily oriented surfaces, Sol. Energy 58 (4-6) (1996) 253-263.

[7] K. Zakšek, T. Podobnikar, K. Oštir, Solar radiation modelling, Comput. Geosci. 31 (2) (2005) 233-240.

[8] T. Statella, E.A.d. Silva, Shadows and clouds detection in high resolution images using mathematical morphology, in: Proceedings of the Pecora 17 Conference "The Future of Land Imaging... Going Operational", Denver, USA, 2008.

[9] A. Fisher, Cloud and cloud-shadow detection in SPOT5 HRG imagery with automated morphological feature extraction, Remote Sens. 6 (2014) 776-800.

[10] S.A. Ackerman, K.I. Strabala, W.P. Menzel, R.A. Frey, C.C. Moeller, L.E. Gumley, Discriminating clear sky from clouds with MODIS, J. Geophys. Res. 103 (D24) (1998) 32141-32157.

[11] Z. Zhu, C.E. Woodcock, Object-based cloud and cloud shadow detection in Landsat imagery, Remote Sens. Environ. 118 (2012) 83-94.

[12] M.C. Rivara, A grid generator based on 4-triangles conforming mesh-refinement algorithms, Int. J. Numer. Methods Eng. 24 (1987) $1343-1354$.

[13] L. Ferragut, R. Montenegro, A. Plaza, Efficient refinement/derefinement algorithm of nested meshes to solve evolution problems, Commun. Numer. Methods Eng. 10 (1994) 403-412.

[14] T. Muneer, Solar radiation model for Europe, Build. Serv. Eng. Res. Technol. 11 (1990) 153-163.

[15] T. Muneer, Solar Radiation and Daylight Models for Energy Efficient Design of Buildings, Architectural Press, Oxford, 1997.

[16] M. Blanco-Muriel, D.C. Alarcón-Padilla, T. López-Moratalla, M. Lara-Coira, Computing the solar vector, Sol. Energy 70 (5) (2001) 431-441.

[17] K. Zanter, LANDSAT-8 (18) Data Users Handbook, Department of the Interior U.S. Geological Survey, Maryland-USA, 2016.

[18] J.C. Jiménez-Muñoz, J.A. Sobrino, D. Skokovic, C. Mattat, J. Cristóbal, Land surface temperature retrieval methods from Landsat-8 thermal infrared sensor data, IEEE Geosci. Remote Sens. Lett. 11 (10) (2014) 1840-1843.

[19] A. Rajeshwari, N.D. Mani, Estimation of land surface temperature of Dindigul district using Landsat 8 data, Int. J. Res. Eng. Technol. 3 (5) (2014) $122-126$.

[20] C.R. Yost, P. Minnis, S. Sun-Mack, Y. Chen, M. McGill, Use of active remote sensors to improve the accuracy of cloud top heights derived from thermal satellite observations, in: Proceedings of the AMS Symposium on Recent Developments in Atmospheric Applications of Radar and Lidar, New Orleans, LA, 2008. January 21-25, CD-ROM, P1.24

[21] T. Inoue, On the temperature and effective emissivity determination of semi-transparent cirrus clouds by bi-spectral measurements in the 10nm window region, J. Meteorol. Soc. Jpn. 63 (1) (1984) 88-99.

[22] T. Chiyu, H. Kon, C. Magono, The cloud-base topography and formation condition of cumulus humilis clouds, J. Fac. Sci. Hokkaido Univ. Ser. 7 Geophys. 4 (1) (1972) 43-57.

[23] J.J. Simpson, J.R. Stitt, A procedure for the detection and removal of cloud shadow from AVHRR data over land, IEEE Trans. Geosci. Remote Sens. 36 (3) (1998) 880-897.

[24] J.P. Musial, F. Husler, M. Sutterlin, C. Neuhaus, S. Wunderle, Probabilistic approach to cloud and snow detection on advanced very high resolution radiometer (AVHRR) imagery, Atmos. Meas. Tech. 7 (2014) 799-822. 